\title{
Buprenorphine improved treatment retention in patients with heroin dependence
}

\author{
Kakko J, Svanborg KD, Kreek MJ, et al. 1-year retention and social function after buprenorphine-assisted relapse \\ prevention treatment for heroin dependence in Sweden: a randomised, placebo-controlled trial. Lancet 2003;361:662-8.
}

\section{QUESTION: In patients with heroin dependence, is buprenorphine added to intensive cognitive behavioural therapy effective?}

\section{Design}

Randomised (allocation concealed*), blinded \{patients, clinicians and data collectors $\} \uparrow, *$ placebo controlled trial with follow up at 1 year.

\section{Setting}

The chemical dependence unit of an addiction centre in Stockholm, Sweden.

\section{Patients}

40 patients $\geq 20$ years of age (mean age $30 \mathrm{y}, 73 \%$ men) with an opiate dependence who were seeking admission for medically assisted heroin withdrawal and had $\geq 1$ year of heroin dependence ( $D S M-I V$ criteria). Exclusion criteria were eligibility for methadone maintenance treatment in Sweden ( $\geq 4$ y of multiple daily heroin use objectively documented in hospital records and $\geq 3$ unsuccessful attempts in abstinence oriented treatment); codependence on alcohol, amphetamines, cannabinoids, or benzodiazepines; neurological disorders; dementia; cognitive impairment; psychosis; or other psychiatric disorder (unless the patient was stable and on treatment not contraindicated to buprenorphine). All patients were included in the analysis.

\section{Intervention}

20 patients were allocated to sublingual buprenorphine, $16 \mathrm{mg}$, for 12 months, with $\geq 6$ months supervised daily administration and possible take home doses thereafter (buprenorphine group). 20 patients were allocated to a tapered 6 day regimen of buprenorphine and then placebo (placebo group). Patients in both groups received 10 weekly group sessions plus 2 booster sessions, in accordance with Marlatt's relapse prevention manual (modified for group therapy with heroin dependent patients), and 45 minute individual counselling sessions every week. All patients provided supervised urine samples 3 times per week.

\section{Main outcome measures}

Retention in treatment at 1 year. Patients were discharged from the study if they failed to attend for $>7$ days or had $>2$ positive urine samples within a 3 month period for any banned substance (ie, $>300 \mathrm{mg} / \mathrm{l}$ for opiates, central stimulants, and cannabinoids and $>100$ $\mathrm{mg} / \mathrm{l}$ for benzodiazepines), unless they complied with more intensive therapy.

Sources of funding:

Swedish Medical

Council and US

National Institutes of

Health.

For correspondence: Dr $M$ Heilig, Karolinska Institute, Stockholm, Sweden. Markus.heilig@ neurotec.ki.se

\section{Main results}

Patients in the buprenorphine group had higher retention in treatment than the placebo group (relative benefit 58.7, 95\% CI 7.4 to 467.4 ): 15 patients in the buprenorphine group were still in treatment at 1 year $(1$ patient dropped out and 4 were involuntarily discharged because of positive urine results) compared with 0 patients in the placebo group (all 20 were involuntarily discharged before 3 months because of positive urine results).

\section{Conclusion}

Buprenorphine added to intensive cognitive behavioural therapy improved treatment retention at 1 year in patients with heroin dependence.

*See glossary.

†Information provided by author.

\section{COMMENTARY}

The study by Kakko et al adds to existing knowledge about the context in which buprenorphine may be a safe and effective treatment for opiate dependence. The strict criteria for admission into methadone maintenance treatment in Sweden, the exclusion of those who qualified for methadone treatment, and the lack of buprenorphine treatment availability in Sweden at the time of the study contribute to the soundness of the study design. This trial used a moderately high dosage of buprenorphine $(16 \mathrm{mg})$ and is one of few that have compared the efficacy of buprenorphine with placebo.

The finding that buprenorphine was superior to placebo will not surprise addiction treatment providers and researchers familiar with this medication. None the less, the $75 \%$ retention rate at 1 year for patients in the buprenorphine group is impressive. Both groups received intensive cognitive behavioural treatment that included individual and group therapy, as well as additional support for patients who relapsed to illicit drug use. As this therapy was so intensive, it would be useful to know if a less intensive cognitive behavioural treatment component, more typical of general clinical practice, would provide comparable results. Additionally, although a fixed daily dose of buprenorphine was used, individualised dose titration based on clinical response might have produced an even better therapeutic outcome.

This study, although small, also found a statistically significant difference in mortality at 1 year between the 2 treatment groups $(0 \%$ in the buprenorphine group $v 20 \%$ in the placebo group, $\mathrm{p}=0.015)$. Although studies generally find higher mortality rates in untreated opiate dependent patients than in patients on opiate maintenance treatment, other studies have not generally found as large a difference as that seen in this study.

Kakko et al highlight the immediate need for effective replacement pharmacotherapy for opiate dependent people who do not meet the strict requirements for methadone treatment in Sweden. Although buprenorphine has been approved for use in Sweden since 1999, it has not been made generally available because of regulatory delays. This study provides compelling evidence that patients would benefit from this treatment if the regulatory gridlock in Sweden could be overcome. The findings also strengthen the international evidence base for the use of buprenorphine in combination with cognitive behavioural therapy.

Paul J Fudala, PhD

VA Medical Center/University of Pennsylvania School of Medicine, Philadelphia, Pennsylvania, USA 